\title{
On the importance of the local interstellar spectrum for the solar modulation parameter
}

\author{
K. Herbst, ${ }^{1}$ A. Kopp, ${ }^{1}$ B. Heber, ${ }^{1}$ F. Steinhilber, ${ }^{2}$ H. Fichtner, ${ }^{3}$ K. Scherer, ${ }^{3}$ \\ and D. Matthiä ${ }^{1,4}$ \\ Received 29 May 2009; revised 1 April 2010; accepted 7 April 2010; published 30 July 2010.
}

[1] Cosmogenic Isotopes are produced in the Earth's atmosphere due to the interaction of galactic cosmic rays with nuclei of atmospheric atoms. Among others, the ${ }^{10} \mathrm{Be}$ concentration in ice cores depends on the galactic cosmic ray flux outside of the Earth's magnetosphere and provides therefore a unique tool to investigate the solar modulation over very long time periods. In this study we investigate the importance of different local interstellar proton spectra often used in literature obtained outside of the Earth's magnetosphere. In order to parameterize the heliospheric modulation we apply the forcefield solution using individual local interstellar proton spectrum (LIS) model dependent $\phi$ values. Thus among atmospheric and magnetospheric processes, the ${ }^{10} \mathrm{Be}$ concentration depends on an interplay of the different LIS and their modulation parameters. Since ${ }^{10} \mathrm{Be}$ measurements do not provide any spectral resolution, PAMELA data have been used for a comparison with the calculated spectra and to provide the model dependent modulation parameters during the solar minimum in July 2006. Within the limitation of the force-field solution and the freedom in parameter space, all LIS lead to a reasonable agreement with the data. Taking the LIS dependency of the modulation parameter into account, we derive linear equations to convert the individual $\phi$ between the different LIS. The conversions used here are then applied to a long-term reconstruction of $\phi$ derived from a record of the cosmogenic radionuclide ${ }^{10} \mathrm{Be}$. By using the derived LIS conversions, we show that the occasionally observed negative $\phi$ values in the reconstruction of Steinhilber et al. (2008) vanish if another LIS model is used. In order to estimate other processes which alter this conclusion, the influence of the palaeo-magnetic field has been included. Thus, if all inner-heliospheric effects on the ${ }^{10} \mathrm{Be}$ flux would be known, this investigation would have the potential to rule out certain LIS.

Citation: Herbst, K., A. Kopp, B. Heber, F. Steinhilber, H. Fichtner, K. Scherer, and D. Matthiä (2010), On the importance of the local interstellar spectrum for the solar modulation parameter, J. Geophys. Res., 115, D00I20, doi:10.1029/2009JD012557.

\section{Introduction}

[2] To study the connection between solar activity, Galactic Cosmic Rays (GCRs), and climate on long timescales, cosmogenic radionuclides like ${ }^{10} \mathrm{Be}$ or ${ }^{14} \mathrm{C}$ play an essential role. In principle cosmogenic radionuclides are produced by the interaction of GCRs with atmospheric nuclei. Before reaching the Earth GCRs are modulated by solar activity and the geomagnetic field [e.g., Beer, 2000; Usoskin and Mursula, 2003; Solanki et al., 2004]. Here we focus on the cosmogenic radionuclide ${ }^{10} \mathrm{Be}$, which is pro-

\footnotetext{
${ }^{1}$ Institut für Experimentelle und Angewandte Physik, ChristianAlbrechts-Universität zu Kiel, Kiel, Germany.

${ }^{2}$ Swiss Federal Institute of Aquatic Science and Technology, Dübendorf, Switzerland.

${ }^{3}$ Theoretische Physik IV, Ruhr-Universität Bochum, Bochum, Germany.

${ }^{4}$ Institut für Luft- und Raumfahrtmedizin, Deutsches Zentrum für Luft- und Raumfahrt, Köln, Germany.

Copyright 2010 by the American Geophysical Union. 0148-0227/10/2009JD012557
}

duced by spallation reactions between secondary neutrons and protons and the atmospheric gases nitrogen and oxygen. Several works [Masarik and Beer, 1999; Webber and Higbie, 2003; McCracken, 2004; McCracken and Beer, 2007; Masarik and Beer, 2009] as well as our results using PLANETOCOSMICS, a GEANT4 based computer code [see Desorgher, 2006], which will be published in a subsequent paper, show that for the ${ }^{10} \mathrm{Be}$ production the most important energy interval of GCR particles lies in the range of 0.4 to $8 \mathrm{GeV} /$ nuc. This energy range corresponds to the interval in which the modulation of the local interstellar proton spectrum (LIS) is strongest [see Meyer et al., 1974].

[3] To describe the modulation of GCRs within the heliosphere we will use the so-called force-field approximation which describes the modulation of GCRs by only a single parameter, the so-called modulation parameter $\phi$. A ${ }^{10} \mathrm{Be}$ data-based reconstruction of this parameter during the past 9300 years mainly from the GRIP ice core [Vonmoos et al., 2006; Steinhilber et al., 2008] shows periods of time, where the force-field approximation results in negative $\phi$ values. If 


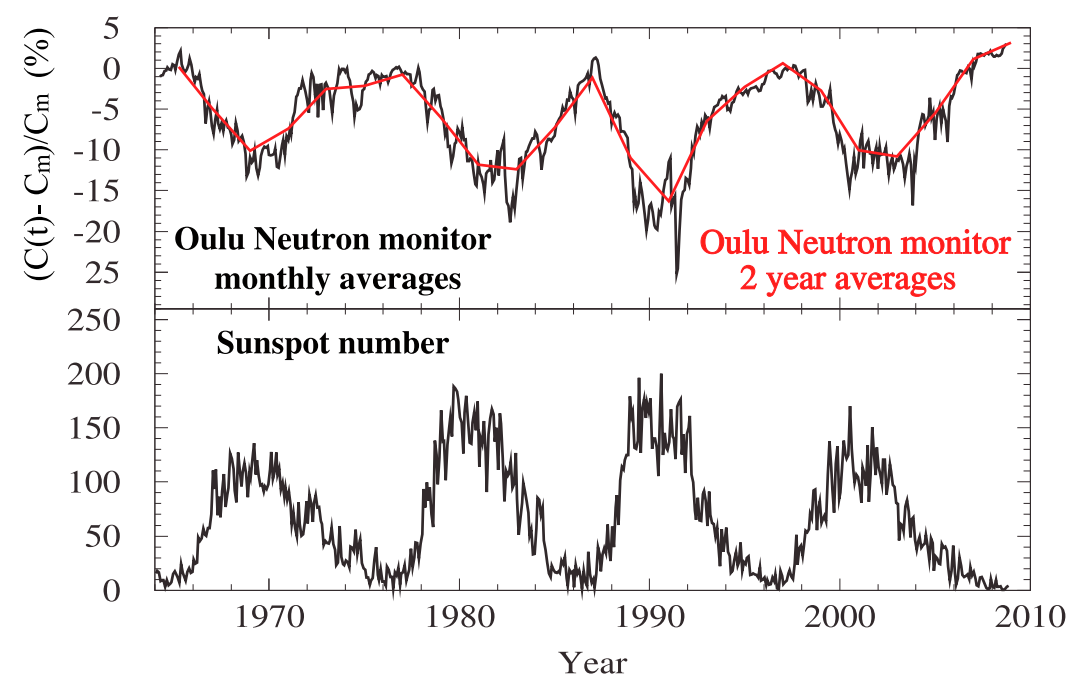

Figure 1. (top) Galactic cosmic ray intensity variation as measured by the Oulu neutron monitor. The quantity $\xi=\left(C(t)-C_{m}\right) / C_{m}$ represents the relative change of the count rate $C(t)$ with respect to the rate $C_{m}=6481$ counts/s, measured during the 1960's solar minimum. (bottom) The sunspot number (SIDC team 2009, http://sidc.oma.be). It is evident that the intensities of GCRs and solar activity are anti-correlated.

this were true, it would imply that the GCR intensity at Earth during these time periods was higher than the unmodulated GCR spectrum at the outer boundary of the heliosphere, called the local interstellar proton spectrum (LIS). This means that a nonphysical acceleration of particles against the solar wind direction had to take place. However, different physical reasons exist, which can explain the existing negative $\phi$ values. These can be either of outer-heliospheric or of inner-heliospheric origin. Uncertainties in the temporal behavior of the geomagnetic field (in particular of its intensity [e.g., Snowball and Muscheler, 2007] as well as its geometry [e.g., Pilchowski et al., 2010]), temporal changes in the transport of ${ }^{10} \mathrm{Be}$ from the atmosphere into the ice (atmospheric mixing), in the ${ }^{10} \mathrm{Be}$ measurements and in the ${ }^{10} \mathrm{Be}$ production calculations for example are of innerheliospheric origin. A possible outer-heliospheric reason could be a temporary enhancement of the LIS due to external processes like supernovae. Another aspect studied in this paper, however, is the choice of the LIS model itself. So far, the LIS has not been directly measured, and LIS models have to be used in the ${ }^{10} \mathrm{Be}$ production calculations. Several LIS models exist in literature, and these differ significantly in the energy ranges below $10 \mathrm{GeV}$. Because the GCRs with energies below $10 \mathrm{GeV}$ are mainly responsible for the ${ }^{10} \mathrm{Be}$ production, an exact knowledge of the LIS is very important.

[4] In this paper we investigate different LIS models with respect to their modulation in the heliosphere. By comparing the various modulated differential proton fluxes with measurements at $1 \mathrm{AU}$ we will show that it is possible to adapt each spectrum to the measurements by using a LIS modeldependent $\phi$ parameter. For this purpose we use here measurements from the PAMELA telescope, a space-borne magnetic spectrometer designed to measure the spectra of anti-protons, positrons, particles and nuclei of the GCRs [see Ambriolia et al., 2003]. For the energy range of 0.4 to $8 \mathrm{GeV} /$ nuc we also investigate the conversion from one modulated spectrum to an other. With this conversions it is possible to study whether or not the choice of the used LIS model is a possible explanation for the negative $\phi$ values.

[5] The paper is structured in the following way: section 2 gives a brief description of the heliospheric transport, while the force-field approximation is discussed more detailed in section 3. The different proton LIS found in literature are compared in section 4 , and our method to convert the modulation parameter $\phi$ between different LIS are described in section 5. Its application to the long-term $\phi$ reconstruction record of Steinhilber et al. [2008] is shown in section 6, and the last section summarizes our results.

\section{Heliospheric Transport}

[6] The intensity of GCRs is modulated as they traverse the turbulent heliospheric magnetic field embedded in the solar wind. The particles are scattered by irregularities in the interplanetary magnetic field and undergo convection as well as adiabatic deceleration in the expanding solar wind. The large-scale heliospheric magnetic field leads to gradient and curvature drifts of CRs in the interplanetary medium. Since all these transport processes depend on the solar magnetic activity, the GCR intensity is anti-correlated with the activity cycle of the Sun. This is shown in Figure 1, displaying the sunspot number in Figure 1, bottom, and the monthly and 2-year averaged cosmic ray variation measured with the Oulu neutron monitor in Figure 1, top. It is evident that the GCR intensity is low when the sunspot number is high and vice versa.

[7] For modeling the measured intensity time-profiles, the transport of cosmic rays in the heliosphere, which was first described by Parkers transport equation [Parker, 1965], has to be taken into account. It consists of different terms:

[8] 1. Outward convection by the solar wind

[9] 2. Gradient and curvature drifts in the global heliospheric magnetic field [Jokipii et al., 1977] 
[10] 3. Diffusion through the irregular heliospheric magnetic field

[11] 4. Adiabatic energy change due to the divergence of the expanding solar wind

[12] 5. Local sources like particles accelerated at the Sun.

[13] The monthly averages of the Oulu neutron monitor (see Figure 1) show alternating "flat" and "peaked" maxima in the time profiles in correlation with the solar magnetic Hale cycle of about 22 years. Today it is generally accepted that these different time profiles are usually an imprint of particle drifts in the global heliospheric magnetic field [e.g., Heber et al., 2006]: in an $A<0$ solar magnetic epoch, like in the 1980's, the intensity of CRs is expected to depend more strongly on the tilt angle of the heliospheric magnetic field. It varies strongly for smaller tilt angles. The intensity in an $A>0$ solar magnetic epoch, on the other hand, should vary significantly less [Potgieter and le Roux, 1992; Heber et al., 2006]. Thus, a detailed description of the neutron monitor observations would rely on a time-dependent numerical solution of Parker's transport equation [see, e.g., Scherer and Ferreira, 2005; Ferreira et al., 2007; Florinski et al., 2008; Florinski and Pogorelov, 2009]. ${ }^{10} \mathrm{Be}$ on the other hand is produced by GCRs in the atmosphere. The timescale of the ${ }^{10} \mathrm{Be}$ transport from the atmosphere, where it is produced, to the ice, where it gets archived, is in the order of 1-2 years. Although on such time scales the modulation of GCRs in the heliosphere still depends on all of the processes described by the Parker equation, a simpler and analytical approximation, called the force-field approximation, is often used in the literature. Although the force-field solution has been used to obtain a parameter to describe solar activity during the space area, we emphasize here that it can not be used in order to describe the details of observed energy spectra in the heliosphere [see Caballero-Lopez and Moraal, 2004].

\section{Force-Field Approximation}

[14] Following Gleeson and Axford [1968] and CaballeroLopez and Moraal [2004], the Parker equation can be reduced to a simple convection-diffusion equation, if (a) there are no additional sources of CRs within the heliosphere, (b) there is a quasi-steady state, (c) the adiabatic energy loss rate is zero, and (d) there is no drift.

[15] If then only the radial direction is taken into account, the Parker equation reduces to

$$
\frac{\partial f}{\partial r}+\frac{v P}{3 \kappa} \frac{\partial f}{\partial P}=0
$$

where $f$ is the cosmic ray distribution function, $r$ is the heliocentric distance, $v$ is the solar wind speed, $P$ is the rigidity of the particle, and $\kappa$ is the diffusion coefficient. If $\kappa$ depends only on $P$ and $r$, a separation in the form of $\kappa(r, P)=\beta \kappa_{1}(r) \kappa_{2}(P)$ can be applied, leading to the following definition of the modulation parameter

$$
\phi(r) \equiv \int_{r}^{r_{b}} \frac{v\left(r^{\prime}\right)}{3 \kappa_{1}} d r^{\prime} .
$$

where the $r_{b}$ is the distance of the outer boundary of the modulation region to the Sun. Note that in this approximation $\kappa_{2}$ is proportional to $\mathrm{P}$. Recent studies however show a much more complex dependence [see Burger et al., 2000].

[16] A typical form of the resulting distribution function is $f=f_{b} \exp \left(-3 \phi /\left(\beta \kappa_{2}\right)\right)$, where $f_{b}$ is the unmodulated spectrum at the outer boundary of the modulation region. Typical values of $\phi$ vary from 300 to $1000 \mathrm{MV}$, for present solar minimum to solar maximum conditions. Note that the force-field formalism results in a modulation potential that causes changes in energy, rigidity, or momentum, while the original assumption was that the adiabatic energy loss term is negligible in comparison with the two spatially streaming terms. There is no straightforward physical reason why this field, force, or energy loss, is related to the true adiabatic loss.

[17] Caballero-Lopez and Moraal [2004] investigated the validity of the force-field model by comparing the results of the force-field solution with those of numerical one dimensional simulations, and they found that the approximation is able to qualitatively describe the modulation of the LIS at $1 \mathrm{AU}$ during the spacecraft era.

[18] The modulation is described by the following equation:

$$
J_{1 A U}(E, \phi)=J_{L I S}(E+\Phi) \frac{(E)\left(E+2 E_{r}\right)}{(E+\Phi)\left(E+\Phi+2 E_{r}\right)}
$$

[19] Here the modulation function $\Phi$ is given by $\Phi=$ $(Z e / A) \phi$ (where $Z$ is the charge and $A$ is the mass number of the cosmic ray particle, respectively and $\phi$ is the modulation parameter in MV. $E$ represents the particles' kinetic energy in (MeV/nuc), $E_{r}$ their rest energy $\left(E_{r} \approx 938 \mathrm{MeV}\right.$ for protons), and $J_{L I S}$ the differential intensity spectrum of the LIS representing the boundary condition of the force-field model.

\section{LIS Proton Spectra}

[20] As mentioned above the force-field model is a first order approximation of the influence of the particle transport in the heliosphere on the GCR spectra, which only depends on the modulation parameter $\phi$ and the input LIS. In the following we will use different proton LIS published in the literature to discuss the influence of the specific LIS on the modulation parameter $\phi$. To allow for an easier comparison between the different published LIS, we transformed all spectra to the same uniform units, giving the energy $E$ in $\mathrm{MeV} /$ nuc, and the differential proton flux $J_{L I S}$ in particles $\mathrm{m}^{-2} \mathrm{~s}^{-1} \mathrm{sr}^{-1}(\mathrm{MeV} / \mathrm{nuc})^{-1}$.

\subsection{Usoskin et al. [2005] Parameterization}

[21] The latitudinal gradients of GCRs as measured by the Ulysses spacecraft [Heber et al., 1996] have been a challenge for transport models until Burger et al. [2000] made a first approach modeling the variation using a two-dimensional computer code solving Parker's transport equation. An equivalent parametrization was found by Usoskin et al. [2005] (US05), which we here have re-written in the uniform units.

$$
J_{U S 05}=\frac{a}{b\left[E\left(E+2 E_{r}\right)\right]^{1.39}+c\left[E\left(E+2 E_{r}\right)\right]^{0.135}},
$$

with $\mathrm{a}=4.157 \cdot 10^{2}, \mathrm{~b}=10^{-7}$ and $\mathrm{c}=1.6488$. 


\subsection{Garcia-Munoz et al. [1975] Parameterization}

[22] We note, that this spectrum has been used in the ${ }^{10} \mathrm{Be}$ production calculations done by Masarik and Beer [1999]. These production calculations have been the basis of Vonmoos et al. [2006] and Steinhilber et al. [2008] for reconstructing the long-term $\phi$ records. Although Masarik and Beer [1999] refer to the LIS by Castagnoli and Lal [1980], they have used GM75, as is pointed out by Masarik and Beer [2009].

$$
J_{G M 75}=a[E+b \exp (c E)]^{-2.65},
$$

with $\mathrm{a}=9.9 \cdot 10^{8}, \mathrm{~b}=780$ and $\mathrm{c}=-2.5 \cdot 10^{-4}$.

\subsection{Webber and Higbie [2003] Parameterization}

[23] This spectrum is derived from galactic cosmic ray propagation calculations by Webber and Lockwood [2001], re-written here in the uniform units.

$$
J_{W H 03}=\left(a E^{2.8}+b E^{1.58}+c E^{0.26}\right)^{-1}
$$

Here $\mathrm{a}=1.89 \cdot 10^{-10}, \mathrm{~b}=5.05 \cdot 10^{-6}$ and $\mathrm{c}=9.33 \cdot 10^{-3}$.

\subsection{Langner et al. [2003] Parameterization}

[24] This LIS, parameterized by Langner et al. [2003] (LA03), is based on a complex GCR propagation model by Moskalenko et al. [2002].

$J_{L A 03}= \begin{cases}\exp \left(a-b(\ln E)^{2}+c \ln E-d \sqrt{E}\right) & E<1000 \mathrm{MeV} / \text { nuc } \\ \exp \left(e-F \ln E-\frac{g}{E}\right) & E \geq 1000 \mathrm{MeV} / \text { nuc }\end{cases}$

Here $\mathrm{a}=0.823, \mathrm{~b}=0.08, \mathrm{c}=1.105, \mathrm{~d}=9.202 \cdot 10^{-2}, \mathrm{e}=$ $22.976, \mathrm{f}=2.86$ and $\mathrm{g}=1.5 \cdot 10^{3}$.

\subsection{Webber and Higbie [2009] Parameterization}

[25] Webber and Higbie [2009] (WH09) have calculated interstellar proton, helium, carbon and iron spectra using a Monte Carlo Diffusion Model (MCDM). Here we parameterize the determined proton LIS (given by Webber and Higbie [2009, Table 1]) the following way:

$$
\begin{aligned}
J_{W H 09}= & \exp \left(a+b \cdot \ln [(\ln (E))]^{2}+c \cdot \sqrt{\ln (E)}+d \cdot(\ln (E))^{-1}\right. \\
& \left.+e \cdot(\ln (E))^{-2}\right)
\end{aligned}
$$

For energies $E<1000 \mathrm{MeV} /$ nuc the parameters are $a=$ $-124.47673, b=-51.83897, c=131.64886, d=-241.72524$, and $e=376.65906$, whereas for energies $E \geq 1000 \mathrm{MeV} /$ nuc $a=0, b=-51.68612, c=103.58884, d=-709.70735$, and $e=1161.55701$.

[26] The accuracy of our parametrization is given in Figure $2 \mathrm{a}$, showing the ratio of our parametrization following equation (8) to the data given by Webber and Higbie [2009]. Our parametrization varies less then $\pm 2.3 \%$ from the published data of Webber and Higbie [2009].

\subsection{Comparison of the Spectra}

[27] Figure 2b shows the ratio of the modulated LIS models of GM75, LA03, WH03 and WH09 to the modulated LIS model of US05 in the energy range of 0.1 to $100 \mathrm{GeV} /$ nuc. As can be seen, these spectra agree quite well with each other for proton energies above $10 \mathrm{GeV} /$ nuc. However, at lower energies differences up to a factor of two exist. As mentioned before, the LIS has not been measured by now and therefore each of these models may approximate it correctly.

[28] Despite the differences all these LIS models allow a parametrization of the modulated spectra in the vicinity of the Earth by using the modulation parameter $\phi$. In order to determine the solar activity parameter, the modulation potential, during recent times we determine the individual $\phi$ values by adjusting the solutions to measurements. This is done by using the proton spectrum during solar minimum conditions in July 2006 observed with the PAMELA instrument [see Casolino et al., 2009, Figure 1], with the energy given in $\mathrm{GeV}$ and the differential proton flux in protons $\left(\mathrm{cm}^{-2} \mathrm{sr}^{-1} \mathrm{~s}^{-1} \mathrm{GeV}^{-1}\right)$. For each LIS an individual value of $\phi$ is needed to describe the observation as best as possible. We found these individual $\phi$ values by minimizing the function

$$
\eta_{i}^{2}=\sum\left(\mathrm{J}_{1 \mathrm{AU}}\left(\mathrm{E}, \phi, \mathrm{LIS}_{\mathrm{i}}\right) / \mathrm{J}_{1 \mathrm{AU}}(\mathrm{E}, \text { PAMELA })-1\right)^{2}
$$

between the PAMELA measurements $\left(\mathrm{J}_{1 \mathrm{AU}}(\mathrm{E}\right.$, PAMELA $\left.)\right)$ and the computed modulated GCR spectra $\left(\mathrm{J}_{1 \mathrm{AU}}\left(\mathrm{E}, \phi, \mathrm{LIS}_{\mathrm{i}}\right)\right)$. The resulting modulation parameters $\phi_{i}$ with corresponding values of $\eta_{i}^{2}$ are listed in Table 1, while the ratio of the adapted spectra to the PAMELA measurements are shown in Figure 2c. Note that the values of $\eta^{2}$ represent the characteristics of the uses LIS models for the energy range of 0.4 to $8 \mathrm{GeV} /$ nuc, the energy range most important for the production of ${ }^{10} \mathrm{Be}$. The LIS model of LA03 shows higher $\eta^{2}$ values then the other models, which is due to high aberrations in the lower energy range, as can bee seen in Figure 2c. Nevertheless, all LIS models, with their individual modulation parameter values are able to represent the observed spectrum fairly well. For example, the US05 spectrum provides a best fit to the measurements with a modulation parameter of $526 \mathrm{MV}$. In the energy range important for the production of ${ }^{10} \mathrm{Be}(0.4$ to $8 \mathrm{GeV} /$ nuc), however, the intensity is underestimated by up to $11 \%$ at an energy of $5140 \mathrm{MeV} /$ nuc. Likewise characteristics can be found for the WH09 model. It provides a modulation parameter $\phi_{W H 09}$ equal $322 \mathrm{MV}$ with a $12 \%$ lower value in this energy range. The only LIS model which overestimates the measured data up to about $8 \%$ at an energy of $2642 \mathrm{MeV} / \mathrm{nuc}$ is the one by Langner et al. [2003], showing a modulation parameter of $749 \mathrm{MV}$.

[29] However, one should keep in mind that the forcefield solution cannot describe the modulation process in all details. The force-field solution e.g. can not reproduce the cross-over of the modulation amplitude, i.e. the intensities are higher for low energy protons in an $\mathrm{A}>0$ than in an $\mathrm{A}<0$ solar magnetic epoch, while the opposite is the case for higher energies, which can be clearly seen in Figure 20 of Heber et al. [2006].

[30] The need of using different $\phi$ values for different LIS emphasizes the importance to always relate the values of $\phi$ to the corresponding LIS model.

\section{Conversion of $\phi$ Between Different LIS}

[31] The results of the former section are taken as motivation to formally describe the conversion between the $\phi$ 

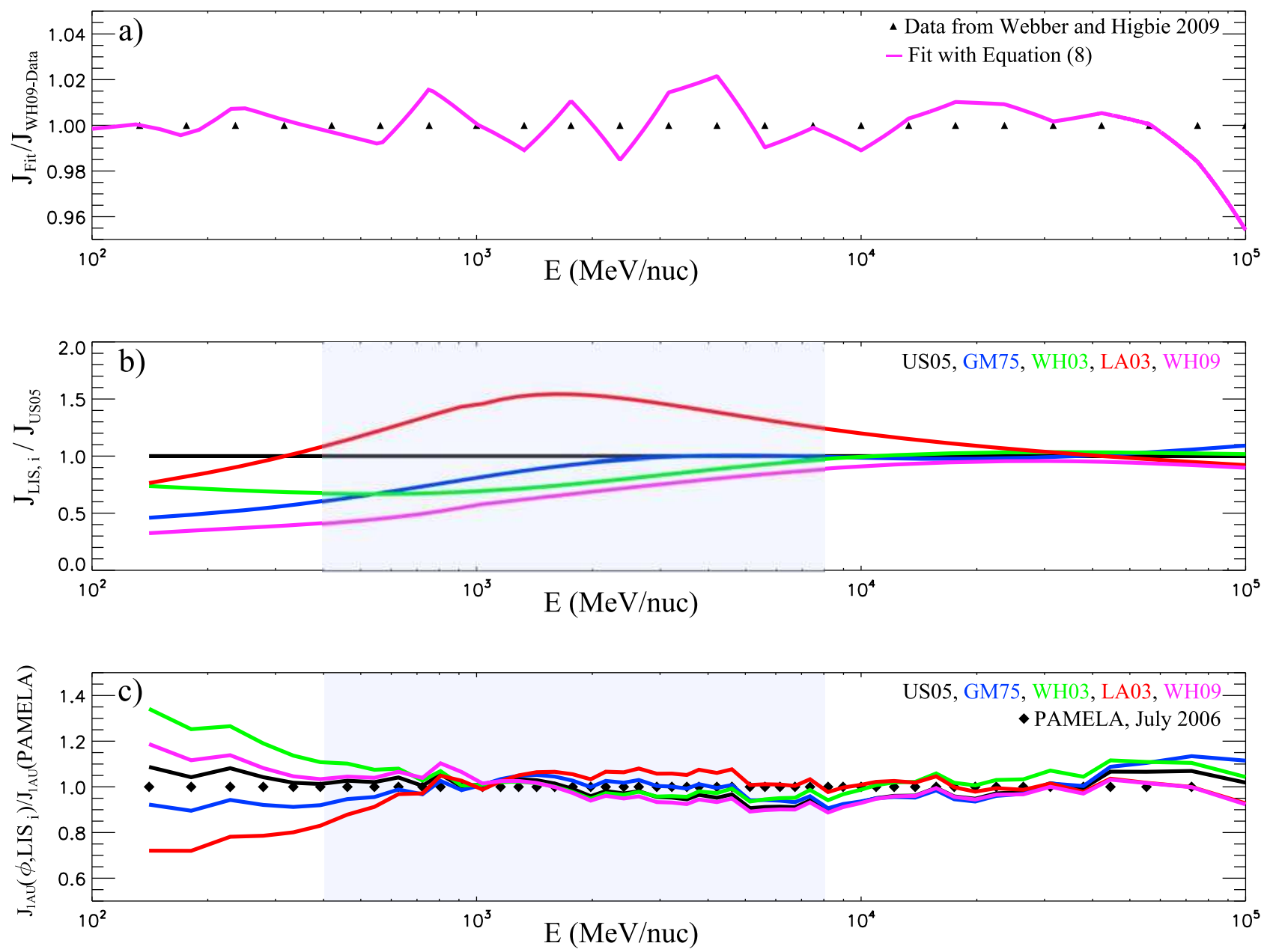

Figure 2. (a) Ratio of our parametrization of the WH09 spectrum (see equation (8), shown here in magenta) to the data given by Webber and Higbie [2009]. A ratio of one is indicated by black triangles. The variation of our parametrization is in the order of $\pm 2.3 \%$. (b) Ratio of the different unmodulated LIS proton spectra used in this study (GM75 (blue), WH03 (green), LA03 (red) and WH09 (magenta)) to US05 (black). Accentuated in light-blue is the energy range relevant for the ${ }^{10} \mathrm{Be}$ production, 0.4 to $8 \mathrm{GeV} /$ nuc. (c) Ratio of the modulated LIS models to the PAMELA measurements in the solar minimum of July 2006 with the same colors as in b). The spectra show strong variations in the low energy range. Nevertheless, in the energy range of 0.4 to $8 \mathrm{GeV} /$ nuc, all spectra are able to reproduce the measurements fairly well with the variations given in the text.

values of the different models. Following the approach first described by Usoskin et al. [2005], the $\phi$ values obtained for the different LIS models can be related to the ones of the modulated Burger et al. [2000] spectrum by linear equations

$$
\phi_{L I S, i}=m_{i} \cdot \phi_{U S 05}+b_{i} .
$$

[32] In order to achieve the coefficients $\mathrm{m}_{i}$ and $\mathrm{b}_{i}$ we apply the following procedure:

[33] 1. For a given $\phi_{U S 05}$ we calculate the spectrum at 1 AU according to equation (3).

[34] 2. The modulated spectrum, $\mathrm{J}_{1 \mathrm{AU}, U S 05}$ is used as reference spectrum in order to fit the other modulated LIS spectra by minimizing the merit function as given in equation (9).
[35] 3. Thus, we generate a paired data set of $\left(\phi_{U S 05}, \phi_{i}\right)$, given in Table 2, which we fit to the model by equation (10) in order to calculate the parameters $b_{i}$ and $m_{i}$.

[36] Our interest lies in the $\phi$ obtained from ${ }^{10} \mathrm{Be}$ data, and thus the energy interval in which the spectra are fitted to

Table 1. Model Dependent Modulation Parameters $\phi_{i}$ Adapted by the Best-Fit Method and the Corresponding $\eta_{i}^{2}$ Values $^{\mathrm{a}}$

\begin{tabular}{lcc}
\hline LIS Models & $\phi$ in MV & $\eta^{2}$ \\
\hline US05 & 526 & 0.059 \\
LA03 & 749 & 0.129 \\
GM75 & 481 & 0.034 \\
WH03 & 378 & 0.040 \\
WH09 & 322 & 0.097 \\
\hline
\end{tabular}

${ }^{a}$ See equation (9). 
Table 2. Selected Values for $\phi_{U S 05}$ in Comparison With the Corresponding $\phi_{L I S, i}$

\begin{tabular}{cccccc}
\hline LIS Models & \multicolumn{5}{c}{$\phi$ in MV } \\
\hline US05 & 200 & 400 & 600 & 800 & 1000 \\
LA03 & 449 & 663 & 876 & 1090 & 1304 \\
GM75 & 159 & 365 & 572 & 779 & 986 \\
WH03 & 57 & 272 & 469 & 666 & 862 \\
WH09 & -2 & 194 & 390 & 585 & 781 \\
\hline
\end{tabular}

each other, has to be similar to the energy interval in which the ${ }^{10} \mathrm{Be}$ production takes place. According to several works [e.g., McCracken, 2004; Masarik and Beer, 2009] as well as our results obtained with the PLANETOCOSMICS code [Desorgher, 2006] the ${ }^{10} \mathrm{Be}$ yield function show that the most important energy interval for the production of ${ }^{10} \mathrm{Be}$ is in the range of 0.4 to $8 \mathrm{GeV} /$ nuc with a production maximum around $1 \mathrm{GeV} /$ nuc. Thus, we will only consider this interval in the $\phi$ conversion. The resulting coefficients $\mathrm{m}_{i}$ and $b_{i}$ are given in Table 3 , and the corresponding linear curves are displayed in Figure 3 (solid lines). The knowledge about which energy interval has to be used, is of great importance. To underline this statement we additionally calculated the conversion coefficients for the extended energy range of 0.1 to $100 \mathrm{GeV} /$ nuc. The results are also shown in Figure 3 (dashed lines). A comparison with the results for the energy range of 0.4 to $8 \mathrm{GeV} /$ nuc show that the conversions are clearly different from each other, so that the choice of the used energy range is indeed of great importance.

\section{Application to a Long-Term Record of $\phi$}

[37] In recent publications [Vonmoos et al., 2006; Steinhilber et al., 2008], the modulation parameter $\phi$ has been reconstructed for the past 9300 years using ${ }^{10} \mathrm{Be}$, mainly measured in the GRIP ice core from Greenland. We note that Vonmoos et al. [2006] and Steinhilber et al. [2008] use the modulation function $\Phi$ in units of $\mathrm{MeV}$. This record is based on the ${ }^{10} \mathrm{Be}$ production calculations of Masarik and Beer [1999]. In these calculations besides protons also alphas and heavier particles have been taken into account merely by increasing the corresponding proton fluxes. This implies that the effect of the different rigidity cutoffs but also the different solar modulation due to the diverse $\mathrm{Z} / \mathrm{A}$ ratios has been neglected. However, the estimated differences are smaller than 10\% [Vonmoos et al., 2006]. Thus, the original $\Phi(\mathrm{MeV})$ record has the meaning of modulation potential $\phi(\mathrm{MV})$.

[38] Figure 4 shows the long-term reconstruction of $\phi$ of Steinhilber et al. [2008]. The left y axis shows the $\phi$ record using the LIS by GM75. As can be seen, for most of the time, the calculated modulation parameter is in the range of presently observed values. However, in some periods (marked as red vertical lines) $\phi$ becomes very small or even negative, for example around the years $500 \mathrm{BP}$ and $1300 \mathrm{BP}$.

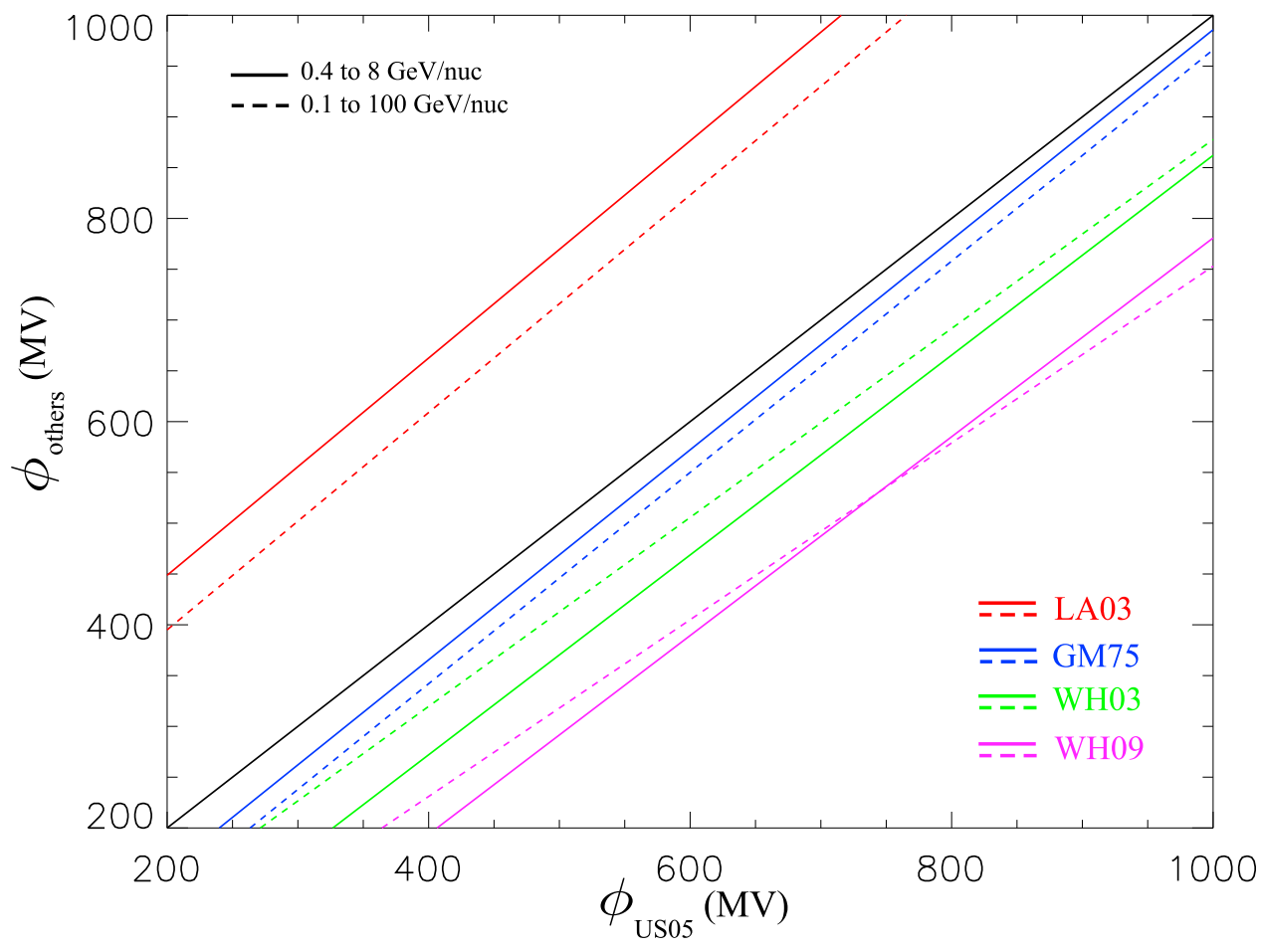

Figure 3. Linear relationship between the different proton LIS models are shown. The $x$ axis represents the modulation parameter of US05, while on the y axis the modulation parameter of the other LIS models is shown. The red lines represent the LA03 LIS, the two blue ones show the GM75 spectrum, green is WH03 and magenta represents WH09. The black line shows US05 itself. Here the solid lines represent the linear equations concerning the energy interval of 0.4 to $8 \mathrm{GeV} /$ nuc, while the dashed lines show the energy range of 0.1 to $100 \mathrm{GeV} /$ nuc. 
Table 3. Parameters $\mathrm{m}_{i}$ and $\mathrm{b}_{i}$ to Convert $\phi$ Obtained With the Different LIS Models to the LIS Model of US05, Using Equation (10)

\begin{tabular}{ccc}
\hline LIS Models & $m_{i}$ & $b_{i}(\mathrm{MV})$ \\
\hline LA03 & 1.06925 & 234.871 \\
GM75 & 1.03378 & -48.0287 \\
WH03 & 0.983462 & -121.360 \\
WH09 & 0.978454 & -197.676 \\
\hline
\end{tabular}

Further periods with negative values are found between 7400 and $5000 \mathrm{BP}$ with the lowest values around $5600 \mathrm{BP}$.

[39] The fact that the modulation parameter reaches zero or even negative values implies that the modulation vanishes. As mentioned earlier, these low values could be interpreted as an acceleration against the solar wind, which is nonphysical.

[40] However, several physical reasons for such negative values exist, which can be divided into outer-heliospheric and inner-heliospheric ones. Inner-heliospheric reasons for the negative $\phi$ values are due to uncertainties in a) the measurement of ${ }^{10} \mathrm{Be}, \mathrm{b}$ ) the geomagnetic field intensity and its geometry in the past, c) temporal changes in the transport of ${ }^{10} \mathrm{Be}$ from the atmosphere into the ice (atmospheric mixing), and $\mathrm{d}$ ) the ${ }^{10} \mathrm{Be}$ production calculations. Besides these inner-heliospheric reasons, also outer-heliospheric ones exist, such as e) the LIS model used in the production calculations, and f) a possible temporal variation of the LIS by additional contributions by e.g. supernovae.
[41] So far no extraordinary events like nearby supernova explosions are known to have taken place during the periods with negative $\phi$ values. Hence, the negative values must be caused by the effects a)-e). Possible reasons for the negative $\phi$ values may be found in the inner-heliospheric effects a) and b), namely the uncertainties in the ${ }^{10} \mathrm{Be}$ measurement and in particular in the palaeo-geomagnetic field intensity reconstruction. As was pointed out by Snowball and Muscheler [2007], there is a large uncertainty in palaeo-geomagnetic field intensity reconstructions. Since the geomagnetic field has to be considered for calculating the $\phi$ values, also the calculated $\phi$ itself holds a large error.

[42] Using the Monte-Carlo technique, Vonmoos et al. [2006] and Steinhilber et al. [2008] found that the error in $\phi$ due to a) the ${ }^{10} \mathrm{Be}$ measurement and $b$ ) the palaeogeomagnetic field varies in the range of 40-150 MV with an average value of $80 \mathrm{MV}$. To investigate this effect, we added the averaged value to the long-term reconstruction providing an upper limit of $\phi$. Since some of the reconstructed modulation potentials are even smaller than $-80 \mathrm{MV}$ this upper limit can not explain all negative $\phi$ values. An uncertainty of over $120 \mathrm{MV}$ instead of the average $80 \mathrm{MV}$ would be necessary to shift all values in a positive range or at least to be consistent with $\phi=0$. Although such negative $\phi$ values could be a consequence of the geomagnetic field, we think that it is not likely that an error $>120 \mathrm{MV}$ was present during all the time periods where negative $\phi$ values are calculated.

[43] Besides the first two inner-heliospheric effects, the other ones, i.e. c), d) and e) might be of importance.

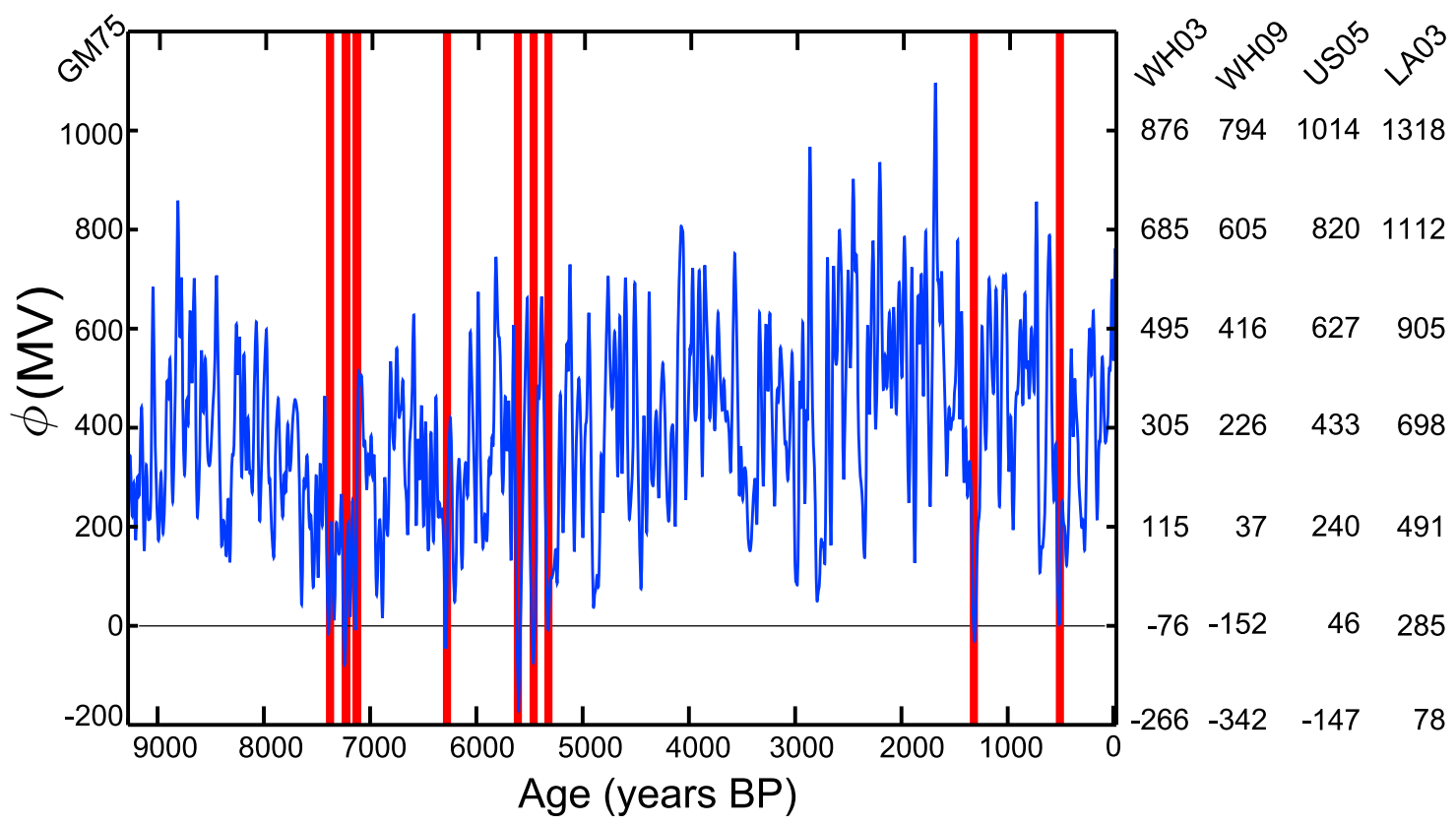

Figure 4. The modulation parameter reconstruction for the past 9300 years based on ${ }^{10} \mathrm{Be}$ mainly measured in the GRIP ice core [see Steinhilber et al., 2008; Vonmoos et al., 2006]. The left y axis show the reconstructed $\phi$ using the GM75 LIS (blue curve). For most time intervals the parameter is in the range of presently observed values, but there are times (marked with the red vertical lines) where the parameter gets zero or even negative (e.g. around the years 500, 1300, and $5600 \mathrm{BP}$ ). The modulation values are converted to the other LIS used in this paper (WH03, LA03, WH03 and WH09), using equation (10). The resulting modulation parameters are shown on the right $\mathrm{y}$-axes. 
[44] In the present work, however, we are concentrating on the possible impacts of outer-heliospheric processes on the reconstruction of the modulation parameter by neglecting the effects c) and d), the influence of the uncertainties in the $10 \mathrm{Be}$ transport and production models. Therefore, the following study is restricted to the heliospheric effect e) which implies that the LIS description itself gives an explanation for the negative values. We note that by neglecting the innerheliospherical effects c) and d) we do not rule them out as possible sources for the negative $\phi$ values at all.

[45] As shown in section 5, $\phi$ itself strongly depends on the used LIS model. It is possible that the LIS model by Garcia-Munoz et al. [1975] (GM75), which has been used for calculating the long-term $\phi$ record, does not represent the true LIS. Now we will demonstrate the importance of the choice of the LIS model and investigate the consequences for the values of the modulation parameter $\phi$.

[46] To study the influence of the LIS, we convert the reconstructed values of $\phi_{G M 75}$ from the long-term $\phi$ record given in Figure 4 (see y axis on the left hand side) to the other LIS models by using the found linear conversions as given in Table 3.

[47] The results for the different LIS are shown on the $y$-axes on the right hand side of Figure 4, showing the corresponding modulation parameters of $\mathrm{WH} 03$, WH09, US05 and LA03, from left to right. The model dependent corresponding $\phi$ values show that the modulation parameter can be shifted to lower or higher values, depending on the used LIS model. So the negative values of $\phi$ occurring in the reconstruction by Vonmoos et al. [2006] and Steinhilber et al. [2008] can either become even more negative by using the LIS models WH03 and WH09, or shift to positive ones and therefore to physically reasonable values of $\phi$ when using the models US05 and in particular LA03.

\section{Summary}

[48] A useful tool to trace the solar activity far back in time are measurements of cosmogenic radionuclides such as ${ }^{14} \mathrm{C}$ and ${ }^{10} \mathrm{Be}$. One essential quantity determining their signal is the intensity of galactic cosmic rays (GCRs) reaching the Earth's orbit. Their intensity is modulated by the solar activity and the geomagnetic field intensity.

[49] As shown by Caballero-Lopez and Moraal [2004], the GCR intensity spectrum at $1 \mathrm{AU}$ can be reasonably well approximated by the force-field solution during the current period of time. In this model, the spectrum at 1 AU only depends on the assumed local interstellar proton spectrum (LIS) and the corresponding modulation parameter $\phi$, describing the solar activity. So far the LIS has not been measured directly, and several estimations of the LIS, called LIS models, exist.

[50] The aim of this paper was the investigation of the importance of the LIS on the modulation parameter $\phi$. Therefore we first compared five different proton LIS models often used in literature showing a variations from each other up to $70 \%$.

[51] By assuming the modulation parameter to be LIS model dependent, we secondly fitted the five modulated LIS models to proton measurements at $1 \mathrm{AU}$ taken with the PAMELA instrument during solar minimum conditions in July 2006. We showed that all used LIS models are able to reproduce the observed PAMELA spectrum fairly well, needing individual LIS model-dependent modulation parameters.

[52] Following the studies of Usoskin et al. [2005] and Steinhilber et al. [2008], we afterwards derived linear equations to convert the LIS model-dependent $\phi$ parameters $\left(\phi_{L I S, i}\right)$ into each other. The works of Masarik and Beer [1999], Webber and Higbie [2003] (WH03), McCracken [2004], McCracken and Beer [2007] and Masarik and Beer [2009] lead to the conclusion that the production of ${ }^{10} \mathrm{Be}$ is most sensitive for variations in the GCR proton flux energy range from 0.4 to $8 \mathrm{GeV} /$ nucleon with a maximum at $1 \mathrm{GeV} /$ nucleon. This energy range corresponds to the range being strongest modulated by solar activity, implying the that ${ }^{10} \mathrm{Be}$ rate is able to reflect the latter. To use the conversion equations with $\phi$ obtained from ${ }^{10} \mathrm{Be}$ measurements later on we fitted the GCR spectra only in the energy range of 0.4 to $8 \mathrm{GeV} /$ nuc. To investigate the importance of the used energy range to the linear conversion equations we also added additional fits using a much wider energy range ( 0.1 to $100 \mathrm{GeV} / \mathrm{nuc}$ ) and showed that the energy range plays an important role.

[53] The found conversion equations in the energy range of 0.4 to $8 \mathrm{GeV} /$ nuc are then applied to the long-term $\phi$ record by Vonmoos et al. [2006] and Steinhilber et al. [2008] based on ${ }^{10} \mathrm{Be}$ mainly from the GRIP ice core. This record (see Figure 4) shows negative $\phi$ values. As was pointed out, such low or even negative values are non-physical, and therefore must be explained. An important source of uncertainty is the palaeo-geomagnetic field intensity reconstruction, which has been considered in the ${ }^{10} \mathrm{Be}$ production calculations of Masarik and Beer [1999], and therewith in $\phi$. We think that it is not likely that all negative phi values could be explained by the uncertainties in the paleo-geomagnetic field. However, other explanations for the negative values exist. Besides the uncertainties in the atmospheric mixing or the ${ }^{10} \mathrm{Be}$ production calculations, also the LIS model itself, which has been used to calculate the ${ }^{10} \mathrm{Be}$ production (here GM75), could be the cause for the negative $\phi$ values. Converting the original long-term $\phi$ record with the derived LIS conversion equations, we found that the LIS models by Langner et al. [2003] and by Burger et al. [2000] would shift the $\phi$ values to positive (physical) values. Whereas using the LIS models by WH03 and WH09 would shift the $\phi$ values to even more negative ones. Thus the long-term reconstruction of the modulation parameter $\phi$ provides the potential to derive the low intensity limits of the LIS. But it has to be kept in mind that first the other sources of uncertainty, i.e. innerheliospheric effects, have to be completely removed from the data.

[54] Acknowledgments. We are grateful for support for the research by the Deutsche Forschungsgemeinschaft (DFG) via the project "Heliocauses" (HE 3279/8-2 and HE 3279/8-3, FI 706/6-2 and FI 706/6-3) carried out within the framework of the DFG priority program 1176 within CAWSES. F. Steinhilber acknowledges financial support by NCCR Climate-Swiss Climate Research.

\section{References}

Ambriolia, M., et al. (2003), PAMELA space mission: The transition radiation detector, 28th International Cosmic Ray Conference, pp. 2121-2124, Univ. Acad. Press, Tokyo. 
Beer, J. (2000), Long-term indirect indices of solar variability, Space Sci. Rev., 94, 53-66.

Burger, R. A., M. S. Potgieter, and B. Heber (2000), Rigidity dependence of cosmic ray proton latitudinal gradients measured by the Ulysses spacecraft: Implications for the diffusion tensor, J. Geophys. Res., 105 , 27,447-27,456, doi:10.1029/2000JA000153.

Caballero-Lopez, R. A., and H. Moraal (2004), Limitations of the force field equation to describe cosmic ray modulation, J. Geophys. Res., 109, A01101, doi:10.1029/2003JA010098.

Casolino, M., et al. (2009), Cosmic ray measurements with Pamela experiment, Nuclear Phys. B, 190, 293-299.

Castagnoli, G. C., and D. Lal (1980), Solar modulation effects in terrestrial production of carbon 14, Radiocarbon, 22, 133-158.

Desorgher, L. (2006), The planetocosmics code, technical report, Univ. Bern, Bern, Switzerland. (Available at http://cosray.unibe.ch/ laurent/ planetocosmics)

Ferreira, S. E. S., M. S. Potgieter, and K. Scherer (2007), Transport and acceleration of anomalous cosmic rays in the inner heliosheath, J. Geophys. Res., 112, A11101, doi:10.1029/2007JA012477.

Florinski, V., and N. V. Pogorelov (2009), Four-dimensional transport of galactic cosmic rays in the outer heliosphere and heliosheath, Astrophys. J., 701, 642-651, doi:10.1088/0004-637X/701/1/642.

Florinski, V., R. B. Decker, and J. A. Le Roux (2008), Low-energy particle acceleration and compression at the termination shock and in the heliosheath, Am. Inst. Phys. Conf. Proc., vol. 1039, pp. 361-366, Am. Inst. of Phys., College Park, Md., doi:10.1063/1.2982472.

Garcia-Munoz, M., G. M. Mason, and J. A. Simpson (1975), The anomalous ${ }^{4} \mathrm{He}$ component in the cosmic-ray spectrum at $\leq 50 \mathrm{MeV}$ per nucleon during 1972-1974, Astrophys. J., 202, 265-275.

Gleeson, L. J., and W. I. Axford (1968), Solar modulation of galactic cosmic rays, Astrophys. J., 154, 1011-1026.

Heber, B., et al. (1996), Spatial variation of $>40 \mathrm{MeV} / \mathrm{n}$ nuclei fluxes observed during the Ulysses rapid latitude scan, Astron. Astrophys. $316,538-546$.

Heber, B., H. Fichtner, and K. Scherer (2006), Solar and heliospheric modulation of galactic cosmic rays, Space Sci. Rev., 125, 81-93, doi:10.1007/ S11214-006-9048-3.

Jokipii, J. R., E. H. Levy, and W. B. Hubbard (1977), Effects of particle drift on cosmic-ray transport: I. General properties, application to solar modulation, Astrophys. J., 213, 861-868.

Langner, U. W., M. S. Potgieter, and W. R. Webber (2003), Modulation of cosmic ray protons in the heliosheath, J. Geophys. Res., 108(A10), 8039, doi:10.1029/2003JA009934

Masarik, J., and J. Beer (1999), Simulation of particle fluxes and cosmogenic nuclide production in the Earth's atmosphere, J. Geophys. Res. 104, 12,099-12,112, doi:10.1029/1998JD200091.

Masarik, J., and J. Beer (2009), An update of particle fluxes and geomagnetic nuclide production in the Earth's atmosphere, J. Geophys. Res. 114, D11103, doi:10.1029/2008JD010557.

McCracken, K. G. (2004), Geomagnetic and atmospheric effects upon the cosmogenic ${ }^{10} \mathrm{Be}$ observed in polar ice, 109, J. Geophys. Res., A04101, doi:10.1029/2003JA010060.

McCracken, K. G., and J. Beer (2007), Long-term changes in the cosmic ray intensity at Earth, 1428-2005, 112, J. Geophys. Res., A10101, doi:10.1029/2006JA012117.

Meyer, P., R. Ramaty, and W. R. Webber (1974), Cosmic rays: Astronomy with energetic particles, Phys. Today, 27, 23-30.
Moskalenko, I. V., A. W. Strong, J. F. Ormes, and M. S. Potgieter (2002), Secondary antiprotons and propagation of cosmic rays in the galaxy and heliosphere, Astrophys. J., 565, 280-296, doi:10.1086/324402.

Parker, E. N. (1965), The passage of energetic charged particles through interplanetary space, Planet. Space Sci., 13, 9-49.

Pilchowski, J., A. Kopp, K. Herbst, and B. Heber (2010), On the definition and calculation of a generalised McIlwain parameter, Astrophys. Space Sci. Trans., 6, 9-17.

Potgieter, M. S., and J. A. le Roux (1992), The simulated features of heliospheric cosmic-ray modulation with a time-dependent drift model: I. General effects of the changing neutral sheet over the period 1985 1990, Astrophys. J., 386, 336-346, doi:10.1086/171020.

Scherer, K., and S. E. S. Ferreira (2005), A heliospheric hybrid model: Hydrodynamic plasma flow and kinetic cosmic ray transport, Astrophys. Space Sci. Trans., 1, 17-27.

Snowball, I., and R. Muscheler (2007), Paleomagnetic intensity data: An Achilles heel of solar activity reconstructions, Holocene, 17, 851-859.

Solanki, S. K., I. G. Usoskin, B. Kromer, M. Schüssler, and J. Beer (2004), Unusual activity of the Sun during recent decades compared to the previous 11,000 years, Nature, 431, 1084-1087, doi:10.1038/nature02995.

Steinhilber, F., J. A. Abreu, and J. Beer (2008), Solar modulation during the Holocene, Astrophys. Space Sci. Trans., 4, 1-6.

Usoskin, I. G., and K. Mursula (2003), Long-term solar cycle evolution: Review of recent developments, Solar Phys., 218, 319-343, doi:10.1023/B:SOLA.0000013049.27106.07.

Usoskin, I. G., K. Alanko-Huotari, G. A. Kovaltsov, and K. Mursula (2005), Heliospheric modulation of cosmic rays: Monthly reconstruction for 1951-2004, J. Geophys. Res., 110, A12108, doi:10.1029/2005JA011250.

Vonmoos, M., J. Beer, and R. Muscheler (2006), Large variations in Holocene solar activity: Constraints from ${ }^{10} \mathrm{Be}$ in the Greenland Ice Core Project ice core, J. Geophys. Res., 111, A10105, doi:10.1029/ 2005JA011500.

Webber, W. R., and P. R. Higbie (2003), Production of cosmogenic Be nuclei in the Earth's atmosphere by cosmic rays: Its dependence on solar modulation and the interstellar cosmic ray spectrum, J. Geophys. Res., 108(A9), 1355, doi:10.1029/2003JA009863.

Webber, W. R., and P. R. Higbie (2009), Galactic propagation of cosmic ray nuclei in a model with an increasing diffusion coefficent at low rigidities: A comparision of the new interstellar spectra with Voyager data in the outer heliosphere, J. Geophys. Res., 114, A02103, doi:10.1029/ 2008JA013689.

Webber, W. R., and J. A. Lockwood (2001), Voyager and Pioneer spacecraft measurements of cosmic ray intensities in the outer heliosphere: Toward a new paradigm for understanding the global solar modulation process: 1. Minimum solar modulation (1987 and 1997), J. Geophys. Res., 106, 29,323-29,332.

H. Fichtner and K. Scherer, Theoretische Physik IV, Ruhr-Universität Bochum, D-44780 Bochum, Germany. (hf@tp4.rub.de; kls@tp4.rub.de)

B. Heber, K. Herbst, and A. Kopp, Institut für Experimentelle und Angewandte Physik, Christian-Albrechts-Universität zu Kiel, D-24118 Kiel, Germany. (heber@physik.uni-kiel.de; herbst@physik.uni-kiel.de; kopp@physik.uni-kiel.de)

D. Matthiä, Institut für Luft- und Raumfahrtmedizin, Deutsches Zentrum für Luft- und Raumfahrt, D-51147 Köln, Germany. (daniel.matthiae@dlr.de)

F. Steinhilber, Swiss Federal Institute of Aquatic Science and Technology, CH-8600 Dübendorf, Switzerland. (friedhelm.steinhilber@eawag.ch) 